Pacific Journal of Mathematics

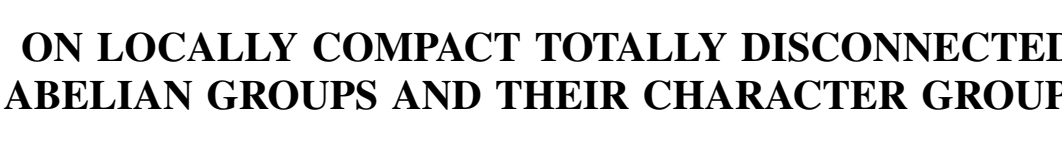




\title{
ON LOCALLY COMPACT TOTALLY DISCONNECTED ABELIAN GROUPS AND THEIR CHARACTER GROUPS
}

\author{
Gerald C. Preston
}

1. Introduction. The Pontrjagin duality theory [4] establishes a dual relationship between pairs of locally compact Abelian groups (referred to in the remainder of the paper as L.C.A. groups): Namely, if two groups are duals each is isomorphic and homeomorphic to the group of all continuous homomorphisms (character group) of the other into the circle $K$, the character group being provided with the compactopen topology. The question arises whether this same property will hold true for groups of mappings into some group other than $K$. Pontrjagin answers this in the negative by showing that if we consider mappings into any group other than $K$, that $K$ itself will not be the character group of its character group. However, for a more restricted class of groups than the class of all L.C.A. groups, the duality property might yet hold true for mappings into some group other than the circle. The purpose of this paper is to show that the duality theorem is valid if we consider mappings of any L.C.A. totally disconnected (L.C.T.D.A.) group into the discrete rationals modulo 1 . It is shown that the same theorem is true using various topologies on this group and a necessary and sufficient condition on the topology is given in order that the theorem be true. For the case of L.C.A. p-primary groups, it is shown that the duality theorem is true using any separated topology on the $p$-primary component of the rationals modulo 1 . Since the rationals modulo 1 are a subgroup of the circle, we are merely using different topologies on the necessary part of the circle, the resulting character groups being the same as those in the classical case. However, for the case of compact T.D.A. groups, a genuinely new theory is exhibited in which the character groups are not in general the same as those of the Pontrjagin theory. Throughout the paper the word group will be used to denote an Abelian separated topological group. The additive notation will be used for the group operation. We shall use the symbol $\simeq$ to represent an algebraic isomorphism and $\cong$ to represent an algebraic isomorphism and topological homeomorphism.

\section{Character theory.}

Definition. Let $G$ and $g$ be two groups. $g$ will be called the Received September 21, 1954. 
auxiliary group. $(G, g)$ will denote the group of all continuous homomorphisms of $G$ into $g$. The topology in $(G, g)$ is as follows : a neighborhood $U$ of 0 in $(G, g)$ will depend on a compact subset $C$ of $G$ and a neighborhood $V$ of 0 in $g$;

$$
U(C, V)=\{\alpha \in(G, g): \alpha(C) \subset V\} .
$$

The proof of the following proposition is omitted.

Proposition 1. $(G, g)$ is a group.

For any $x \in G, x$ may be considered to define a homomorphism $\bar{x}$ of $(G, g)$ into $g$ as follows :

$$
\bar{x}(\alpha)=\alpha(x) \text { for any } \alpha \in(G, g) .
$$

Under this definition, $\bar{x}$ is a continuous homomorphism of $(G, g)$ into $g$. The proof of the following two propositions for the case that $g=K$ are given in [4] and carry over exactly to the general case.

Proposition 2. If $G$ is a L.C.A. group and has sufficiently many continuous homomorphisms into $g$, that is, given $x \neq 0$ in $G$, there is an $\alpha \in(G, g)$ such that $\alpha(x) \neq 0$, then a one-to-one continuous image of $G$ is included in $((G, g), g)$.

Proposition 3. $\quad\left(G_{1} \times G_{2}, g\right) \cong\left(G_{1}, g\right) \times\left(G_{2}, g\right)$.

By induction,

$$
\left(\prod_{1}^{n} G_{i}, g\right) \cong \prod_{1}^{n}\left(G_{i}, g\right) \text {. }
$$

In a similar manner, the following proposition is easily shown.

Proposition 4. $\quad\left(G, g_{1} \times g_{2}\right) \cong\left(G, g_{1}\right) \times\left(G, g_{2}\right)$ and by induction,

$$
\left(G, \prod_{1}^{n} g_{i}\right) \cong \prod_{1}^{n}\left(G, g_{i}\right)
$$

Definition. Let $H$ be a subgroup of $G . H^{*} \subset(G, g)$ is defined as follows :

$$
H^{*}=\{\alpha \in(G, g): \alpha(H)=\{0\}\}
$$

The following proposition can be proved directly.

Proposition 5. $H^{*}$ is a closed subgroup of $(G, g)$. 
Since the next theorem applies to open compact subgroups of $G$, we note that in a L.C.T.D.A. group, any neighborhood of 0 contains an open compact subgroup [5].

THEOREM 2.1. If $H$ is an open compact subgroup of $G$, then $(G / H, g) \cong H^{*}$.

Proof. Let $A \in(G \mid H, g)$. We define $\alpha \in H^{*}$ as follows: if $N$ represents the natural mapping of $G$ onto $G / H$ then $\alpha(x)=A(N(x))$. We now map $A \stackrel{f}{\rightarrow} \alpha$. It is shown in [4] for the case $g=K$, that $f$ is a continuous, onto, isomorphism. The proof carries over to the general case. It remains to show $f^{-1}$ is continuous. Let $U^{\prime}=U^{\prime}(D, V)$ be any neighborhood of 0 in $(G / H, g)$. Since $H$ is open, $G / H$ is discrete and hence $D$ is finite. Let

$$
D=\left(x_{1}+H, x_{2}+H, \cdots, x_{n}+H\right) .
$$

If $C=N^{-1}(D), C \subset G$, then

$$
C=\left(x_{1}+H \cup x_{2}+H \cup \cdots \cup x_{n}+H\right) .
$$

Since each coset $x_{i}+H$ is compact, $C$ is compact. Letting $U_{H^{*}}=U(C, V) \cap H^{*}$ (a neighborhood of 0 in $H^{*}$ ), we have $f^{-1}\left(U_{H^{*}}\right) \subset U^{\prime}$ and $f^{-1}$ is continuous.

THEOREM 2.2. If $g$ is discrete and $G$ is a L.C.A. group, then $(G, g) \cong(\hat{g}, \widehat{G})$, where $\hat{G}$ and $\hat{g}$ are the classical character groups.

Proof. Consider any $\alpha \in(G, g)$. Define $\hat{\alpha} \in(\hat{g}, \widehat{G})$ as follows [6]:

$$
\hat{\alpha}(\hat{x})(X)=\hat{x}(\alpha(X)) \quad \hat{x} \in \hat{g}, X \in G .
$$

$\hat{\alpha}(\hat{x})$ is defined since $\alpha$ and $\hat{x}$ are both continuous homomorphisms. In [1] and [6] it is shown that $\hat{\alpha}$ is a continuous homomorphism of $\hat{g}$ into $\hat{G}$. We map $\alpha \stackrel{f}{\rightarrow} \hat{\alpha} . \quad f$ is obviously a homomorphism and is one-to-one since $g$ has sufficiently many characters in the classical theory. By considering $\widehat{\alpha}$ of $\widehat{G}$ into $\widehat{g}$ defined similarly to $\hat{\alpha}$ it is easily seen that $f$ is onto. Since in $(G, g)$ we may consider neighborhoods of the type $U(C,\{0\})$ it is easy to verify that $f$ is bicontinuous.

We note here that if $g$ is discrete and $G$ is compact, $(G, g)$ is discrete since $U(G,\{0\})=\{0\}$ is open in $(G, g)$.

Definition. A group $G$ is said to be divisible if for any $x \in G$ and any integer $n$, there is a $y \in G$ such that $n y=x$.

THEOREM 2.3. If $g$ is divisible and discrete and $H$ is an oxen 
compact subgroup of $G$ then $(H, g) \cong(G, g) / H^{*}$.

Proof. Let $\beta_{H} \in(H, g)$. It is shown in [6] that since $g$ is divisible, $\beta_{H}$ may be extended to a continuous homomorphism $\beta$ of $(G, g)$. If $\beta_{H}$ has two extensions, their difference is 0 on $H$ so they are in the same coset of $H^{*}$. If $\bar{\beta}$ is the coset of $H^{*}$ containing $\beta$, we map $\stackrel{f}{\rightarrow}_{\rightarrow} \bar{\beta} . f$ is clearly an isomorphism of $(H, g)$ onto $(G, g) / H^{*}$. By a remark above, $(H, g)$ is discrete. But $H^{*}=U(H,\{0\})$ which is open so that $(G, g) / H^{*}$ is also discrete.

3. p-primary groups. A theorem of Braconnier [1] shows that the $p$-primary groups are the basic elements in the structure of L.C.T.D.A. groups. We present this theorem after giving the necessary definitions.

Definition. A group $G$ is said to be $p$-primary if for any $x \in G$, the mapping $n \rightarrow n x$ of the integers into $G$ may be extended continuously to the mapping $z \rightarrow z x$ of the $p$-adic integers, $Z_{p}$ into $G$.

Definition. Let $G_{\lambda}, \lambda \in I$, be a family of topological groups and $H_{\lambda}$ be open normal subgroups of the $G_{\lambda}$. Let $G=\prod_{\lambda \in I} G_{\lambda}$ and

$$
G^{\prime}=\left\{x=\left\{x_{\lambda}\right\} \in G: x_{\lambda} \in H_{\lambda} \text { for all but a finite number of } \lambda\right\} .
$$

$G^{\prime}$ is provided with the following topology: $U$ is a neighborhood of 0 in $G^{\prime}$ if $U$ is a neighborhood of 0 in II $H_{\lambda}$. Under these conditions, $G^{\prime}$ is a topological group and is called the local direct product of $G_{\lambda}$ with respect to $H_{\lambda}$ we write

$$
G^{\prime}=\mathrm{L} \Pi G_{\lambda}: H_{\lambda} .
$$

$G^{\prime}$ is L.C. if and only if each $G_{\lambda}$ is L.C. and all but a finite number of $H_{\lambda}$ are compact.

Definition. Let $G$ be a group and

$G_{p}=\{x \in G$ : the mapping $n \rightarrow n x$ of the integers into $G$ may be extended continuously to the mapping $z \rightarrow z x$ of the $p$-adic integers into $G\}$.

$G_{p}$ is called the $p$-primary component of $G$. If $G$ is a L.C.T.D.A. group then $G_{p}$ is a closed subgroup of $G$.

Theorem 3.1 [1]. Let $G$ be a L.C.T.D.A. group and $H$ any open compact subgroup of $G$. Then $G$ is isomorphic to the local direct product of its primary components $G_{p}$ relative to primary components $H_{p}=H \cap G_{p}$ of $H$. If $G$ is compact then $G=\Pi G_{p}$. 
Using the notation $C_{p^{n}}=$ cyclic group of order $p^{n}, Q_{p}=p$-adic numbers, $Z_{p}=p$-adic integers, and $p^{\infty}=Q_{p} \mid Z_{p}$, we note that the following simple examples show that with $p^{\infty}$ as auxiliary group, a duality theorem obtains for L.C.A. $p$-primary groups of rank 1 (see [1] or [2]) :

$$
\left(C_{p}, p^{\infty}\right) \cong C_{p},\left(Z_{p}, p^{\infty}\right) \cong p^{\infty},\left(Q_{p}, p^{\infty}\right) \cong Q_{p}, \text { and }\left(p^{\infty}, p^{\infty}\right) \cong Z_{p} .
$$

Proposition 3 shows that the duality theorem holds for groups of any finite rank $n$. We shall show in the present section that if $p^{\infty}$ is used as an auxiliary group, a duality theory obtains for all L.C.A. $p$-primary groups. This theorem is implied by a theorem in [2] but our proof is given from anentirely different point of view. We shall represent $Q_{p}$ as all sums $\left\{\sum_{-N}^{\infty} a_{i} p^{i}, 0 \leqq a_{i}<p\right\}, Z_{p}$ as sums $\left\{\sum_{0}^{\infty} a_{i} p^{i}, 0 \leqq a_{i}<p\right\}$, and $p^{\infty}$ as all sums $\left\{\sum_{-N}^{-1} a_{i} p^{i}, 0 \leqq a_{i}<p\right\}$.

THeOREM 3.2. If $g$ is any $p$-primary group then $\left(Z_{p}, g\right) \simeq g$. Furthermore $\left(Z_{p}, g\right)$ has a stronger topology than $g$, that is, the identity mapping. of $\left(Z_{p}, g\right)$ onto $g$ is continuous.

Proof. Let $\alpha \in\left(Z_{p}, g\right)$ and map $\alpha \stackrel{f}{\rightarrow} \alpha(1) \in g$. We show that $f$ is a continuous isomorphism of $\left(Z_{p}, g\right)$ onto $g . \quad f$ is obviously a homomorphism. Since $g$ is $p$-primary, $z x$ is defined for any $z \in Z_{p}$ and $x \in g$. If $\alpha(1)=0$ then $\alpha(n)=0$ so that $\alpha$ is 0 . Thus $f$ is one-to-one. Now given $x \in g$, define $\alpha(z)=z x . \quad \alpha$ is clearly a homomorphism and is continuous by a theorem in [1]. Also $\alpha(1)=x$. Hence $f$ is onto. Finally, given any neighborhood $V$ of 0 in $g$ we have

$$
f(U(\{1\}, V)) \subset V
$$

so that $f$ is continuous.

THEOREM 3.3. If $g$ is a L.C.A. p-primary group then $\left(Z_{p}, g\right) \cong g$.

Proof. Continuing in the notation of the proof of Theorem 3.2, we show that $f$ is bicontinuous. Let $U(C, V)$ be a neighborhood of 0 in $\left(Z_{p}, g\right)$. Without loss of generality, we may consider the neighborhood $U\left(Z_{p}, V\right)$ since $Z_{p}$ is compact. Since $g$ is a L.C.A. $p$-primary group, $g$ is T.D. and there exists an open subgroup $H$ such that $H \subset V$. Now if $\alpha \in f^{-1}(H)$ then $\alpha(1) \in H, \alpha(n) \in H$ and since $H$ is closed, $\alpha\left(Z_{p}\right) \subset H \subset V$. That is, $\alpha \in U\left(Z_{p}, V\right)$. Hence $f^{-1}(H) \subset U\left(Z_{p}, V\right)$ and since $H$ is open $f^{-1}$ is continuous.

THeorem 3.4. If $G$ is a L.C.A. p-primary group then $\left(\left(G, p^{\infty}\right), p^{\infty}\right)$ $\cong G$. 
Proof. $\left(G, p^{\infty}\right) \cong\left(\hat{p^{\infty}}, \widehat{G}\right)$ by Theorem 2.2 since $p^{\infty}$ is discrete. $\cong\left(Z_{p}, \widehat{G}\right)$ since it is well known that $\widehat{p^{\infty}} \cong Z_{p}$. $\cong \widehat{G}$ by Theorem 3.3 since $\widehat{G}$ is always L.C.A. $p$-primary with $G[1]$.

Therefore,

$$
\begin{aligned}
\left(\widehat{G}, p^{\infty}\right) & \cong\left(\widehat{p^{\infty}}, \widehat{G}\right) \\
& \cong\left(Z_{p}, G\right) \text { as } \hat{G} \cong G \\
& \cong G .
\end{aligned}
$$

We next note that the circle $K$ contains a subgroup isomorphic to $p^{\infty}$ but with non-discrete topology, namely, the subgroup of all elements of order $p^{n}$. We shall call this subgroup $p^{\infty k}$. We note from the proof of Theorem 3.4 that $\left(G, p^{\infty}\right) \cong \widehat{G} \cong\left(G, p^{\infty k}\right)$. Thus for the $K$ topology and the discrete topology, we obtain the same character groups. We shall show that the identical theory obtains with the use of $p^{\infty}$ provided with any separated topology.

LEMMA 3.1. Let $p^{\infty \tau}$ be the algebraic group $p^{\infty}$ with separated topology $\tau$. If $\alpha$ is a continuous homomorphism of a L.C.A. group $G$ into $p^{\infty \tau}$, then $\alpha$ is a continuous mapping of $G$ into the discrete group $p^{\infty}$.

Proof. Let $H$ be an open compact subgroup of $G$ and consider $\alpha(H)$ which is also compact. Since the only locally compact topology on a countable group is discrete, (see the footnote to the proof of Theorem 3.6) $\alpha(H) \neq p^{\infty \tau}$. Since the only proper subgroups of $p^{\infty}$ are finite, $\alpha(H)$ is finite and hence discrete. Thus $\alpha$ is continuous on $H$ to $p^{\infty}$ and since $H$ is open, $\alpha$ is continuous on $G$ to $p^{\infty}$.

LeMma 3.2. For any separated topology $\tau$, there exists in $p^{\infty r} a$ neighborhood $V$ of 0 such that $V$ contains no nonzero subgroups.

Proof. Since the only proper subgroups of $p^{\infty}$ are the groups isomorphic to $C_{p^{n}}$, any nonzero subgroup contains the elements $p^{-1}, 2 p^{-1}$, $\cdots,(p-1) p^{-1}$. However, since $\tau$ is separated, there is a neighborhood of zero excluding all these elements.

Theorem 3.5. If $G$ is a L.C.A. p-primary group then $\left(G, p^{\infty}\right) \cong\left(G, p^{\infty \tau}\right)$.

Proof. The isomorphism follows from Lemma 3.1. If $G$ is compact then by Lemma 3.2, using $V$ as there defined, $(G, V)=\{0\}$ is open so 
that $\left(G, p^{\infty \tau}\right)$ is discrete. Also $\left(G, p^{\infty}\right)$ is discrete so the theorem holds for compact groups. Now consider the case where $G$ is discrete. We may consider $\left(G, p^{\infty \tau}\right)$ and $\left(G, p^{\infty}\right)$ as the same group with different topologies. With this consideration, the identity mapping of $\left(G, p^{\infty}\right)$ onto $\left(G, p^{\infty \tau}\right)$ is clearly continuous since any set open in $\left(G, p^{\infty \tau}\right)$ will be open in $\left(G, p^{\infty}\right)$. Since $\left(G, p^{\infty}\right)$ is compact, the identity mapping is bicontinuous and the theorem is true when $G$ is discrete. Now let $G$ be a L.C.A. group and $H$ an open compact subgroup of $G$. We know that $\left(G \mid H, p^{\infty}\right)$ $\cong H^{*}$ by Theorem 2.1. Since $H$ is open, $G / H$ is discrete and $H^{*}$ is a compact subgroup of $\left(G, p^{\infty \tau}\right) . \quad H^{*}$ is also open since $H^{*}=U(H, V)$ where $V$ is the neighborhood of Lemma 3.2. Thus $H^{*}$ is an open compact subgroup of $\left(G, p^{\infty r}\right)$. Now since $H^{*}$ is compact in both $\left(G, p^{\infty \tau}\right)$ and $\left(G, p^{\infty}\right)$, the identity mapping of $\left(G, p^{\infty}\right)$ onto $\left(G, p^{\infty}\right)$ is bicontinuous on $H^{*}$. But since $H^{*}$ is open in both topologies, the mapping is bicontinuous on $\left(G, p^{\infty}\right)$ to $\left(G, p^{\infty \tau}\right)$.

Corollary. $\left(\left(G, p^{\infty \tau}\right), p^{\infty \tau}\right) \cong G$.

Proof. $\quad\left(\left(G, p^{\infty \tau}\right), p^{\infty \tau}\right) \cong\left(\left(G, p^{\infty}\right), p^{\infty \tau}\right) \cong\left(\left(G, p^{\infty}\right), p^{\infty}\right) \cong G$.

The question remains as to whether any group other than $p^{\infty}$ will give a similar duality theory when used as the auxiliary group. Although the question is not completely settled, we shall present a theorem which limits the type of group which may be considered. First we prove a preliminary lemma.

LEMMA 3.3. If $g$ is a group with arbitrarily small open subgroups and if $H$ is a discrete divisible subgroup of $g$, then $H$ is a direct factor.

Proof. Since $H$ is discrete, there is a neighborhood $V$ of 0 in $g$ such that $V \cap H=\{0\}$. By hypothesis, $V$ contains an open subgroup $K_{1}$ and $K_{1} \cap H=\{0\}$. Now the set of open subgroups $K_{\lambda} \supset K_{1}$ with the property that $K_{\lambda} \cap H=\{0\}$, ordered by inclusion clearly satisfies the hypothesis of Zorn's lemma. Hence we may assert the existence of a maximal open subgroup $K_{0} \supset K_{1}$ whose intersection with $H$ is 0 . The fact that $H+K_{0}=g$ follows from a standard argument.

For any $p$-primary group $g$, we let $g^{\infty}$ be the subgroup of $g$ consisting of all elements of finite order and infinite height. From [1], the group $g^{\infty}$ with the discrete topology, call it $g^{\infty}$, must be a direct product of groups isomorphic to $p^{\infty}$. Hence $g^{\infty} \cong \Pi p^{\infty}$ with some appropriate topology.

THEOREM 3.6. Let $g$ be a p-primary group such that for any L.C.A. p-primary group $G,((G, g), g) \cong G$. Then 
(a) if $(G, g)$ is L.C.A. then $g \simeq p^{\infty}$,

(b) if $g$ is L.C.A. then $g \cong p^{\infty}$,

(c) if $g \neq p^{\infty}$ then $g^{\infty} \simeq \Pi p^{\infty}$ with infinitely many factors and $g$ contains arbitrarily small compact nonopen subgroups.

Proof. (a) We first observe that since the only homomorphic images of $p^{\infty}$ are $p^{\infty}$ itself or $\{0\}$, that $g$ must contain a subgroup $p^{\infty \tau} \simeq p^{\infty}$ in order that there be sufficiently many mappings of $p^{\infty}$ into $g$. Now in the notation of the proof of Theorem 3.2, $\left(Z_{p}, g\right) \simeq g$ under the mapping $\alpha \stackrel{f}{\rightarrow} \alpha(1)$. We show next that $f^{-1}\left(p^{\infty \tau}\right) \cong p^{\infty}$, that is, $p^{\infty}$ with the discrete topology is a subgroup of $\left(Z_{p}, g\right)$. By Lemma 3.2, there is a neighborhood $V$ of 0 in $g$ such that $V \cap p^{\infty \tau}$ contains no nonzero subgroup of $p^{\infty \tau}$. Consider the neighborhood $U\left(Z_{p}, V\right)$ of 0 in $\left(Z_{p}, g\right)$. If $\alpha \in U\left(Z_{p}, V\right)$, then $\alpha(1) \in V$. Now if $\alpha(1) \in p^{\infty \tau}$, then $\alpha\left(Z_{p}\right) \subset p^{\infty \tau}$ since for sufficiently large $n, \alpha\left(p^{n}\right)=0$. But $\alpha\left(Z_{p}\right)$ is a subgroup, so that $\alpha\left(Z_{p}\right)=\{0\}$. Thus if $\alpha \in U\left(Z_{p}, V\right) \cap f^{-1}\left(\underline{p}^{\infty \tau}\right)$, that is, $\alpha(1) \in V \cap p^{\infty \tau}, \alpha=0$. Thus $U\left(Z_{n}, V\right) \cap$ $f^{-1}\left(p^{\infty \tau}\right)=\{0\}$ and $f^{-1}\left(\underline{p}^{\infty \tau}\right)$ is discrete. Thus by Lemma 3.3, $p^{\infty}$ is a direct factor of $\left(Z_{p}, g\right)$, let $\left(Z_{p}, g\right)=p^{\infty} \times g_{1}$. Now $\left(\left(Z_{p}, g\right), g\right)=\left(p^{\infty} \times g_{1}, g\right)$ $\cong\left(p^{\infty}, g\right) \times\left(g_{1}, g\right)$ so that by hypothesis, $Z_{p} \cong\left(p^{\infty}, g\right) \times\left(g_{1}, g\right)$. We note that one of these factors must be $\{0\}$ since if $Z_{p}$ were expressible as a direct product $p^{\infty} \cong\left(Z_{p}, p^{\infty}\right)$ would be also. Clearly if $g_{1} \subsetneq\{0\},\left(g_{1}, g\right)$ $\neq\{0\}$. Therefore since $\left(p^{\infty}, g\right) \neq\{0\}, g_{1}=\{0\}$ and $\left(Z_{p}, g\right) \cong p^{\infty}$. Thus since $\left(Z_{p}, g\right) \simeq g, g \simeq p^{\infty}$. It follows from the method of the proof that if $g$ contains $p^{\infty \tau}$ as a direct factor, $\mathrm{g}=p^{\infty \tau}$.

(b) In the case that $g$ is L.C.A. $\left(Z_{p}, g\right) \simeq g$ by Theorem 3.3. Hence $\left(Z_{p}, g\right)$ is L.C.A. $p$-primary and exactly as in the proof of part (a), $g \simeq p^{\infty}$. Since the discrete topology is the only locally compact topology on a countable group ${ }^{1}, g \cong p^{\infty}$.

(c) Assume that $g^{\infty} \simeq p^{\infty} \times p^{\infty} \times \cdots \times p^{\infty}, n$ factors, $n>1$. We note first that $\left(p^{\infty}, g\right) \simeq\left(p^{\infty}, g^{\infty}\right)$. Since $p^{\infty}$ is discrete, we have $\left(p^{\infty}, g^{\infty}\right) \simeq$ all homomorphisms of $p^{\infty}$ into $\left.g^{\infty}\right\}$, and $\left(p^{\infty}, g^{\infty}\right) \simeq\left(p^{\infty}, g^{\infty}\right)$. But $\left(p^{\infty}, g^{\infty \pi}\right) \cong$ $\left(p^{\infty}, p^{\infty} \times p^{\infty} \times \cdots \times p^{\infty}\right) \cong\left(Z_{p} \times Z_{p} \times \cdots \times Z_{p}\right)$ which is compact. Thus the algebraic isomorphism of $\left(p^{\infty}, g^{\infty d}\right)$ into $\left(p^{\infty}, g^{\infty}\right)$ is continuous and one-toone, hence bicontinuous. Therefore $\left(p^{\infty}, g^{\infty}\right) \cong Z_{p} \times Z_{p} \times \cdots \times Z_{p}$. $\left(\left(p^{\infty}, g\right), g\right)$ $\cong\left(Z_{p}, g\right) \times\left(Z_{p}, g\right) \times \cdots \times\left(Z_{p}, g\right) \simeq g \times g \times \cdots \times g$ which is clearly not $p^{\infty}$. Hence if $g \nsubseteq p^{\infty}, g^{\infty}$ must contain infinitely many factors. Now if $g$ contains small open compact subgroups, $g$ will be L.C.A and by part (b) $g \cong p^{\infty}$. We assume that $g$ contains a neighborhood $V$ which contains no compact subgroups. In this case $\left(Z_{p}, g\right)$ is discrete since $U\left(Z_{p}, V\right)=\{0\}$ is open. Now if $g \neq p^{\infty}$, then

${ }^{1}$ This statement follows from the fact that a non-discrete locally compact countable space must have isolated points. See, for example [3]. 


$$
\left(Z_{p}, g\right) \cong p^{\infty} \times g_{1}
$$

since a divisible subgroup of a discrete group is a direct factor. Hence

$$
Z_{p} \cong\left(\left(Z_{p}, g\right), g\right) \cong\left(p^{\infty} \times g_{1}, g\right) \cong\left(p^{\infty}, g\right) \times\left(g_{1}, g\right)
$$

which is a contradiction as observed above. Hence every neighborhood of $g$ must contain a compact nonopen subgroup.

This theorem shows that a reasonable conjecture for a possible auxiliary group would be $\Pi p^{\infty}$ or wk $\Pi p^{\infty}$ (see $\S 5$ for definition) provided with a connected or at least non-T.D. topology. We also know from Theorem 3.6 that if a duality theorem obtains, it is entirely different from the classical type in that the duals of some compact groups (in particular $Z_{p}$ ) will be non-locally compact.

4. A duality theory for compact T.D.A. groups. We present in this section a duality theory which does not carry over to the general L.C.A. case but applies to all compact T.D.A. groups. The theory is of some interest since the character groups are genuinely different from those in the Pontrjagin theory. The auxiliary group will be $g=\Pi_{1}^{\infty} p_{i}^{\infty} \tau_{i}$ where $p_{1}, p_{2}, \cdots$ represent all primes and the $\tau_{i}$ are any separated topologies, $\Pi_{1}^{\infty} p_{i}^{\infty} \tau_{i}$ being the full direct product with Tychonoff topology.

LEMMA 4.1. If $G$ is $p_{k}$-primary, then $(G, g) \cong\left(G, p_{k}^{\infty} \tau_{k}\right)$.

Proof. Since a group may be $p$-primary for at most one $p$, it is clear that if $\alpha \in(G, g)$, then

$$
\alpha(G) \subset\left(0, \cdots, 0, p_{k}^{\infty \tau} \tau_{k}, \cdots\right) .
$$

Thus $(G, g) \cong\left(G, p_{k}^{\infty \tau_{k}}\right)$.

Since we deal with direct products in the following theorems, we shall adopt the convention that we do not distinguish between the elements $x_{\lambda} \in G_{\lambda}$ and $\left(0, \cdots, 0, x_{\lambda}, 0, \cdots\right) \in \Pi G_{\lambda}$.

Lemma 4.2. If $G_{i}, i=1,2, \cdots$, are L.C.A. $p_{i}$-primary groups, then

$$
\left(\prod_{1}^{\infty} G_{i}, g\right) \cong \Pi_{1}^{\infty}\left(G_{i}, p_{i}^{\infty \tau_{i}}\right) \text {. }
$$

Proof. Let $G=\prod_{1}^{\infty} G_{i}$ and $A \subseteq(G, g)$. We determine $\left\{\alpha_{i}\right\} \in \prod_{1}^{\infty}\left(G_{i}, p_{i}^{\infty \tau_{i}}\right)$ where $\alpha_{i} \in\left(G_{i}, p_{i}^{\infty} \tau_{i}\right)$ as follows: $\alpha_{i}\left(x_{i}\right)=A\left(x_{i}\right)$. Thus each $\alpha_{i}$ is a continuous homomorphism of $G_{i}$ into $p_{i}^{\infty \tau_{i}}$. We know $\alpha_{i}\left(G_{i}\right) \subset p_{i}^{\infty} \tau_{i}$ from Lemma 4.1. We shall map $\stackrel{\stackrel{f}{\rightarrow}}{\rightarrow}\left\{\alpha_{i}\right\}$ and show that $f$ is a bicontinuous 
isomorphism of $(G, g)$ onto $\Pi_{1}^{\infty}\left(G_{i}, p_{\imath}^{\infty} \tau_{i}\right) . \quad f$ is clearly a homomorphism. Since $A\left(\left\{x_{i}\right\}\right)=\left\{A\left(x_{i}\right)\right\}, f$ is one-to-one. If $\left\{\alpha_{i}\right\} \in \Pi_{1}^{\infty}\left(G_{i}, p_{i}^{\infty \tau_{i}}\right)$, we may define $A\left(\left\{x_{i}\right\}\right)=\left\{\alpha_{i}\left(x_{i}\right)\right\}$. It is easily seen that $A \in(G, g)$ and that $f(A)$ $=\left\{\alpha_{i}\right\}$ so that $f$ is onto. We must now prove that $f$ is bicontinuous. If $W$ is a neighborhood of 0 in $\Pi_{1}^{\infty}\left(G_{i}, p_{i}^{\infty} \tau_{i}\right)$, then $W$ is of the form

$$
\left(\cdots, W_{i_{1}}\left(C_{i_{1}}, V_{i_{1}}\right), \cdots, W_{i_{n}}\left(C_{i_{n}}, V_{i_{n}}\right), \cdots\right)
$$

where $W_{i_{j}} \subset\left(G_{i_{j}}, p_{i_{j}}^{\infty \tau_{i}}\right)$. If we let

$$
D=\left(0, \cdots, C_{i_{1}}, \cdots, C_{i_{n}}, 0, \cdots\right) \subset \prod_{1}^{\infty} G_{i},
$$

then $D$ is compact. Letting $V=\left(\cdots, V_{i_{1}}, \cdots, V_{i_{n}}, \cdots\right)$, a neighborhood of 0 in $g$, it is easy to verify that $f(U(D, V)) \subset W$. Now let $U(D, V)$ be a neighborhood of 0 in $\left(\Pi_{1}^{\infty} G_{i}, g\right)$ where $V=\left(\cdots, V_{i_{1}}, \cdots, V_{i_{n}}, \cdots\right) \subset g$. If $F_{i}$ is the projection of $G$ onto $G_{i}$, let $C_{i_{j}}=F_{i_{j}}(D)$ for $j=1, \cdots, n$. Then

$$
W=\left(\cdots, W_{i_{1}}\left(C_{i_{1}}, V_{i_{1}}\right), \cdots, W_{i_{n}}\left(C_{i_{n}}, V_{i_{n}}\right), \cdots\right)
$$

is a neighborhood of 0 in $\prod_{1}^{\infty}\left(G_{i}, p_{i}^{\infty \tau_{i}}\right)$ with the property that $f^{-1}(W) \subset U$. Thus $f$ is bicontinuous.

THEOREM 4.1. If $G$ is a compact T.D.A. group, then $((G, g), g) \cong G$.

Proof. First we note that $G=\Pi_{1}^{\infty} G_{i}$ where the $G_{i}$ are the $p_{i}$-primary components of $G$ and that $\left(G_{i}, p_{i}^{\infty \tau_{i}}\right)$ is a L.C.A. $p$-primary group. Hence by Lemma 4.2 and Theorem 3.4 we have

$$
\begin{aligned}
&\left.((G, g), g) \cong\left(\left(\prod_{1}^{\infty} G_{i}, g\right), g\right) \cong\left(\prod_{1}^{\infty} G_{i}, p_{i}^{\infty \tau_{i}}\right), g\right) \cong \prod_{1}^{\infty}\left(\left(G_{i}, p_{i}^{\infty \tau_{i}}\right), p_{i}^{\infty \tau_{i}}\right) \\
& \cong \prod_{1}^{\infty} G_{i}=G .
\end{aligned}
$$

In the classical theory, $(G, g)$ is discrete if $G$ is compact. However, in this case,

$$
(G, g)=\prod_{1}^{\infty}\left(G_{i}, p_{i}^{\infty} \tau_{i}\right)
$$

which is an infinite product of discrete groups since each $G_{i}$ is compact and hence each $\left(G_{i}, p_{i}^{\infty} \tau_{i}\right)$ is discrete. Such a product is clearly not discrete so that these character groups of some compact T.D.A. groups are not the same as the classical character groups. 
5. A duality theory for all L.C.T.D.A. groups. The auxiliary group in this case will be the discrete weak direct product of $p_{i}^{\infty}$ for all prime $p_{i}, g=\mathrm{wk} \mathrm{II}_{1}^{\infty} p_{i}^{\infty}$, that is the subgroup of the full direct product in which all but a finite number of components are 0 . Our object is to prove that $((G, g), g) \cong G$ for all L.C.T.D.A. groups. We shall use the following notation throughout all lemmas and theorems of the present section. $G$ will be a L.C.T.D.A. group. From Theorem 3.1, $G=$ $\mathrm{L} \Pi G_{p}: H_{p}$ where the $G_{p}$ are the primary components of $G$ and the $H_{p}$ are the primary components of some open compact subgroup $H \subset G . F_{p}$ will be the projection of $G$ onto $G_{p} . \quad g^{d}=$ wk $\Pi_{1}^{\infty} p_{i}^{\infty}$ with discrete topology and $g^{\tau}=\mathrm{wk} \Pi_{1}^{\infty} p_{i}^{\infty}$ with topology $\tau$.

LEMMA 5.1. If $G$ is a $p_{k}$-primarg group, then $\left(G, g^{d}\right) \cong\left(G, p_{k}^{\infty}\right)$.

Proof. The proof is the same as that of Lemma 4.1.

LemMA 5.2. If $C$ is a compact subset of $G$, then $F_{p}(C) \subset H_{p}$ for all but a finite number of the p's.

Proof. Consider the discrete group $G / H$. Let $D$ be the image of $C$ under the natural mapping of $G$ onto $G / H . \quad D$ is compact and hence finite. Let

$$
D=\left\{a_{1}+H, a_{2}+H, \cdots, a_{n}+H\right\},
$$

and let $a_{j}=\left\{a_{p}^{j}\right\}$. Since $G$ is a local direct product, for any $1 \leqq j \leqq n$, $a_{p}^{j} \in H_{p}$ for all but a finite number of the $p$ 's. Let $p_{i_{1}}, \cdots, p_{i_{n}}$ be the set of indices for which $a_{p}^{j} \notin H_{p}$ for some $1 \leqq j \leqq n$. Now for any $x \in C$ we have $x \in a_{k}+H$ for some $1 \leqq k \leqq n$. Thus $x=a_{k}+h, h \in H$ and $x_{p}=a_{p}^{k}+h_{p}$, $h_{p} \in H_{p}$. For $p \neq p_{i_{1}}, \cdots, p_{i_{n}}$ we have $a_{p}^{k} \in H_{p}$ and hence $x_{p} \in H_{p}$ so that $F_{p}(C) \subset H_{p}$.

Before stating the next theorem, we note that since $H_{p}^{*} \cong\left(G_{p} / H_{p}, p^{\infty}\right)$, the group $H_{p}^{*}$ is an open compact subgroup of $G_{p}$ for all $p$.

THEOREM 5.1. If $G=\mathrm{LII} G_{p}: H_{p}$, then

$$
\left(G, g^{d}\right) \cong \operatorname{L\Pi }\left(G_{p}, p^{\infty}\right): H_{p}^{*} .
$$

Proof. Let

$$
A \in\left(\mathrm{LI} G_{p}: H_{p}, g^{d}\right)
$$

be given. For each $p$ we determine $\alpha_{p} \in\left(G_{p}, p^{\infty}\right)$ as follows : 


$$
\alpha_{p}\left(x_{p}\right)=A\left(x_{p}\right) \text { for } x_{p} \in G_{p} .
$$

Each $\alpha_{p}$ is clearly a continuous homomorphism. We must show that all but a finite number are in their respective $H_{p}^{*}$ 's. If $\alpha \notin H_{p}^{*}$ for some infinite set $S$ of $p$ 's, then for each $p \in S$, let $h_{p} \in H_{p}$ be such that $\alpha_{p}\left(h_{p}\right) \neq 0$ and let

$$
X=\left\{x_{p}\right\} \in \operatorname{L\Pi } G_{p}: H_{p}
$$

where $x_{p}=h_{p}$ for $p \in S, x_{p}=0$ otherwise. Now

$$
\lim _{n \rightarrow \infty} \sum_{p_{1}}^{p_{n}} x_{p}=X
$$

so that

$$
\lim _{n \rightarrow \infty} A\left(\sum_{p_{1}}^{p_{n}} x_{p}\right)=A(X)
$$

But $A\left(x_{p}\right)=\alpha_{p}\left(x_{p}\right) \neq 0$ for an infinite set of $p$ 's and therefore $A\left(\left\{x_{p}\right\}\right) \notin g^{a}$ which is a weak direct product. Hence $S$ must be finite. Now we map

$$
A \in\left(\mathrm{L} \Pi G_{p}: H_{p}, g^{a}\right) \stackrel{\jmath}{\rightarrow}\left\{\alpha_{p}\right\} \in \mathrm{L} \Pi\left(G_{p}, p^{\infty}\right): H_{p}^{*} .
$$

$f$ is an isomorphism as in the proof of Lemma 4.2. If

$$
\left\{\alpha_{p}\right\} \in \mathrm{L} \Pi\left(G_{p}, p^{\infty}\right): H_{p}^{*},
$$

define $A\left(\left\{x_{p}\right\}\right)=\left\{\alpha_{p}\left(x_{p}\right)\right\} \in g^{d}$. If $p_{i_{1}}, \cdots, p_{i_{n}}$ is the set of $p$ 's such that $\alpha_{p} \notin H_{p}^{*}$, we observe that the neighborhood $U=\left(\cdots, V_{p_{i_{1}}}, \cdots, V_{p_{i_{n}}}, \cdots\right)$ where $\alpha_{p_{t_{j}}}\left(V_{p_{i_{j}}}\right)=\{0\}$ and $V_{p_{i}} \subset H_{p_{i_{j}}}$ is such that $A(U)=\{0\}$ so that $A$ is a continuous homomorphism. Since $f(A)=\left\{\alpha_{p}\right\}, f$ is onto. It remains to show the homeomorphism of the two groups. If

$$
U=\left(\cdots, U_{p_{i_{1}}}\left(C_{p_{i_{1}}},\{0\}\right) \cap H_{p_{i_{1}}}^{*}, \cdots, U_{p_{i_{n}}}\left(C_{p_{i_{n}}},\{0\}\right) \cap H_{p_{i_{n}}}^{*}, \cdots\right)
$$

is any neighborhood of 0 in $\operatorname{L\Pi }\left(G_{p}, p^{\infty}\right): H_{p}^{*}$ we may consider $C_{p_{i j}}$ as a compact subset of Lח $G_{p}: H_{p}$. If $D=\bigcup_{1}^{n} C_{p_{i_{j}}}$ and $W=W(D \cup H,\{0\})$ then $f(W) \subset U$. Now if $U(D,\{0\})$ is any neighborhood of 0 in (Lח $G_{p}$ : $\left.H_{p}, g^{d}\right)$ we let $C_{p}=F_{p}(D)$ and by Lemma 5.2 we may let $p_{i_{1}}, \cdots, p_{i_{n}}$ be the set of $p$ 's such that $C_{p_{i j}} \varangle H_{p_{i j}}$. It is easily verified that

$$
V=\left(\cdots, V_{p_{i_{1}}}\left(C_{p_{i_{1}}} \cup H_{p_{i_{1}}},\{0\}\right), \cdots, V_{p_{i_{n}}}\left(C_{p_{i_{n}}} \cup H_{p_{i_{n}}},\{0\}\right), \cdots\right)
$$

is such that $f^{-1}(V) \subset U$.

THEOREM 5.2. If $G$ is L.C.T.D.A., then $\left(\left(G, g^{d}\right), g^{d}\right) \cong G$. 
Proof. $\quad\left(\left(G, g^{d}\right), g^{d}\right) \cong\left(\left(\mathrm{L} \Pi G_{p}: H_{p}, g^{d}\right), g^{d}\right) \cong\left(\mathrm{L} \Pi\left(G_{p}, p^{\infty}\right): H_{p}^{*}, g^{d}\right)$. Clearly $\left(G_{p}, p^{\infty}\right)$ is $p$-primary and is the $p$-primary component of $\operatorname{L\Pi }\left(G_{p}\right.$, $\left.p^{\infty}\right): H_{p}^{*}$. Also, $H_{p}^{*}$ is an open compact subgroup of $\left(G_{p}, p^{\infty}\right)$ as noted in the statement preceding Theorem 5.1. Applying Theorem 5.1 to the group LII $\left(G_{p}, p^{\infty}\right): H_{p}^{*}$, we have

$$
\left(\left(G, g^{a}\right), g^{d}\right) \cong \operatorname{LII}\left(\left(G_{p}, p^{\infty}\right), p^{\infty}\right):\left(H_{p}^{*}\right)^{*} .
$$

Since by Theorem 3.4, $\left(\left(G_{p}, p^{\infty}\right), p^{\infty}\right) \cong G_{p}$, it will be sufficient to show that $\left(H_{p}^{*}\right)^{*}=H_{p}$. Now from Theorem 2.1, $H_{p}^{*} \cong\left(G_{p} / H_{p}, p^{\infty}\right)$. Applying this result to the open compact subgroup $H_{p}^{*}$ of $\left(G_{p}, p^{\infty}\right)$, we obtain

$$
\left(H_{p}^{*}\right)^{*} \cong\left(\left(G_{p}, p^{\infty}\right) / H_{p}^{*}, p^{\infty}\right) \text {. }
$$

But from Theorem 2.3 we have

$$
\left(G_{p}, p^{\infty}\right) / H_{p}^{*} \cong\left(H_{p}, p^{\infty}\right) .
$$

Therefore

$$
\left(H_{p}^{*}\right)^{*} \cong\left(\left(H_{p}, p^{\infty}\right), p^{\infty}\right) \cong H_{p}
$$

since $H_{p}$ is a compact $p$-primary group.

We next investigate the conditions on the topology $\tau$ which give us a duality theorem with $g^{\tau}$ as the auxiliary group.

Lemma 5.3. If $A$ is a continuous homomorphism of a L.C.T.D.A. group $G$ into $g^{\tau}$, then $A$ is a continuous homomorphism of $G$ into $g^{d}$.

Proof. Let $H$ be an open compact subgroup of $G$. Consider the compact group $A(H)$ in $g^{\tau}$. Since the only locally compact topology on a countable group is discrete, (see proof of Theorem 3.6) $A(H)$ is discrete and hence $A^{-1}(0)$ is open in $H$. Therefore, $A$ is a continuous mapping on $H$ to $g^{a}$. But since $H$ is open, $A$ is continuous on $G$ to $g^{a}$.

THeOREM 5.3. $\left(G, g^{i}\right) \simeq\left(G, g^{\tau}\right)$ for any topology $\tau$. If $G$ is discrete then $\left(G, g^{d}\right) \cong\left(G, g^{\tau}\right)$.

Proof. Lemma 5.3 establishes the algebraic isomorphism between $\left(G, g^{d}\right)$ and $\left(G, g^{\tau}\right)$. It is clear that if $G$ is discrete, then $\left(G, g^{d}\right)$ is compact and therefore $\left(G, g^{d}\right) \cong\left(G, g^{\tau}\right)$, since $\left(G, g^{d}\right)$ has a stronger topology than $\left(G, g^{\tau}\right)$.

THEOREM 5.4. A necessary and sufficient condition that $\left(\left(G, g^{\tau}\right), g^{\tau}\right)$ $\cong G$ for all L.C.T.D.A. groups $G$ is that $g^{\tau}$ not have arbitrarily small subgroups. 
Proof. Since $\left(G, g^{d}\right) \simeq\left(G, g^{\tau}\right)$, if $g^{\tau}$ does not have arbitrarily small subgroups the proof that $\left(G, g^{d}\right) \cong\left(G, g^{\tau}\right)$ proceeds exactly as the proof of Theorem 3.5. Thus the sufficiency of the condition follows from the duality theorem for $g^{a}$. To show the necessity, we consider the discrete group

$$
G=\mathrm{wk} \prod_{1}^{\infty} C_{p_{i}}=\mathrm{L} \Pi C_{p_{i}}:\{0\} .
$$

From Theorem 5.3 we have $\left(G, g^{\tau}\right) \cong\left(G, g^{d}\right)$, and from Theorem 5.1

$$
\left(G, g^{d}\right) \cong \mathrm{L} \Pi C_{p_{i}}: C_{p_{i}} \cong \prod_{1}^{\infty} C_{p_{i}} .
$$

Thus if we assume $\left(\left(G, g^{\tau}\right), g^{\tau}\right) \cong g$, we have

$$
\left(\prod_{1}^{\infty} C_{p_{i}}, g^{\tau}\right) \cong \mathrm{wk} \prod_{1}^{\infty} C_{p_{i}} .
$$

However, if $g^{\tau}$ has arbitrarily small subgroups it has small finite subgroups and hence small copies of cyclic groups of prime order. Thus every neighborhood of 0 in $g$ will contain a continuous homomorphic nonzero image of $\Pi_{1}^{\infty} C_{p_{i}}$ and $\left(\Pi_{1}^{\infty} C p_{i}, g^{\tau}\right)$ cannot be discrete.

We note that if $G$ is compact, then $\left(\left(G, g^{\tau}\right), g^{\tau}\right) \cong G$ for any topology $\tau$. If $G$ is compact, then $\left(G, g^{d}\right)$ is discrete so every homomorphism of $\left(G, g^{d}\right)$ into $g^{d}$ is continuous. Hence if $y \in\left(\left(G, g^{\tau}\right), g^{\tau}\right)$, then $y$ may be considered as belonging to $\left(\left(G, g^{d}\right), g^{a}\right) \cong G$. Thus we see that the embedding of $G$ in $\left(\left(G, g^{\tau}\right), g^{\tau}\right)$ described in Proposition 2 of $\S 2$ covers $\left(\left(G, g^{\tau}\right), g^{\tau}\right)$. Since $G$ is compact and the mapping of $G$ onto $\left(\left(G, g^{\tau}\right), g^{\tau}\right)$ is continuous and one-to-one, it is bicontinuous and the duality theorem is true if $G$ is compact.

\section{REFERENCES}

1. J. Braconnier, Sur les groupes topologiques localement compacts, J. Math. Pures Appl., 27 (1948), 1-85.

2. I. Kaplansky, Dual modules over a valuation ring, Proc. Amer. Math. Soc., 4 (1953), 213-219.

3. R. L. Moore, Fundations of point set theory, Amer. Math. Soc. Colloquium Publ. vol. 13, New York, 1932.

4. L. Pontrjagin, To'pological groups, Princeton University Press, Princeton, 1939.

5. E. R. Van Kampen, Locally bicompact Abelian groups and their character groups, Ann. of Math., 36 (1935), 448-463.

6. A. Weil, L'intégration dans les groupes topologiques et ses applications, Paris, 1938.

Purdue University and San Jose State College 


\section{PACIFIC JOURNAL OF MATHEMATICS}

\section{EDITORS}

H. L. Royden

Stanford University

Stanford, California

E. HewitT

University of Washington

Seattle 5 , Washington
R. P. Dilworth

California Institute of Technology Pasadena 4, California

E. G. Straus

University of California

Los Angeles 24, California

\section{ASSOCIATE EDITORS}

E. F. BECKENBACH

C. E. BURGESS

H. BUSEMANN

H. FEDERER

\author{
M. HALL \\ P. R. HALMOS \\ V. GANAPATHY IYER \\ R. D. JAMES
}

M. S. KNEBELMAN

I. NIVEN

T. G. OSTROM

M. M. SCHIFFER
J. J. STOKER

G. SZEKERES

F. WOLF

K. YOSIDA

\section{SUPPORTING INSTITUTIONS}

UNIVERSITY OF BRITISH COLUMBIA

CALIFORNIA INSTITUTE OF TECHNOLOGY

UNIVERSITY OF CALIFORNIA

MONTANA STATE UNIVERSITY

UNIVERSITY OF NEVADA

OREGON STATE COLLEGE

UNIVERSITY OF OREGON

UNIVERSITY OF SOUTHERN CALIFORNIA
STANFORD UNIVERSITY

UNIVERSITY OF UTAH

WASHINGTON STATE COLLEGE

UNIVERSITY OF WASHINGTON

AMERICAN MATHEMATICAL SOCIETY CALIFORNIA RESEARCH CORPORATION HUGHES AIRCRAFT COMPANY 


\section{Pacific Journal of Mathematics}

\section{Vol. 6, No. $1 \quad$ November, 1956}

David Blackwell, An analog of the minimax theorem for vector payoffs..... 1

L. W. Cohen, A non-archimedian measure in the space of real

sequences ..................................... 9

George Bernard Dantzig, Constructive proof of the Min-Max theorem ..... 25

Jim Douglas, On the numerical integration of quasilinear parabolic

differential equations ............................... 35

James Michael Gardner Fell, A note on abstract measure ............. 43

Isidore Isaac Hirschman, Jr., A note on orthogonal systems . . . . . . . . . . 47

Frank Harary, On the number of dissimilar line-subgraphs of a given

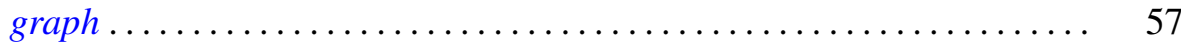

Newton Seymour Hawley, Complex bundles with Abelian group......... 65

Alan Jerome Hoffman, Morris Newman, Ernst Gabor Straus and Olga

Taussky, On the number of absolute points of a correlation ...........

Ernst Gabor Straus and Olga Taussky, Remark on the preceding paper.

Algebraic equations satisfied by roots of natural numbers . . ........ 97

Ralph D. James, Summable trigonometric series ................. 99

Gerald R. Mac Lane, Limits of rational functions . . . . . . . . . . . . . . . 111

F. Oberhettinger, Note on the Lerch zeta function ................. 117

Gerald C. Preston, On locally compact totally disconnected Abelian groups and their character groups ........................... 121

Vikramaditya Singh and W. J. Thron, On the number of singular points, located on the unit circle, of certain functions represented by

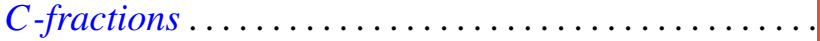

Sherman K. Stein, The symmetry function in a convex body ... 145 Edwin Weiss, Boundedness in topological rings.............

Albert Leon Whiteman, A sum connected with the series for the partition

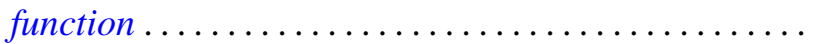

Alfred B. Willcox, Some structure theorems for a class of Banach algebras.

Joseph Lawrence Zemmer, Some remarks on p-rings and their Boolean geometry... 